MATEC Web of Conferences 49, 01003 (2016)

DOI: $10.1051 /$ matecconf/20164901003

(C) Owned by the authors, published by EDP Sciences, 2016

\title{
Effect of Surfactant Concentration in the Emulsions on the Process of Oleophilic Porous Structures Imbibition
}

\author{
Olga S. Shtyka ${ }^{a}$, Jerzy P. Sęk \\ Lodz University of Technology, Poland
}

\begin{abstract}
The spontaneous imbibition has been a subject of the scientific interest being a background process for numerous industrial technologies and occurring in the natural environment. In literature the experimental and theoretical results regarding this phenomenon describe a media imbibition with single-phase liquids and the relation between the process rate and media characteristics. The imbibition of oleophilic porous structures with two-phase liquids, only one phase of which was wetting, is an objective of the current publication. The main purpose is to estimate the influence of both surfactant fraction and the dispersed phase concentration on the mentioned process. The imbibition rate was investigated during model experiments with stabilized oil-in-water emulsions having the dispersed phase concentrations of $10 \mathrm{vol} \%, 30 \mathrm{vol} \%$ and $50 \mathrm{vol} \%$. The prepared emulsions differed with fraction of the added surfactant, i.e. $1 \mathrm{vol} \%, 2 \mathrm{vol} \%$ and $5 \mathrm{vol} \%$. The obtained results allowed to conclude that at the $h_{i m} \geq 0.02 \mathrm{~m}$, the dispersed phase concentration and viscosity decreased versus height. However, the raise of the surfactant fraction caused the increase of mass and height of the imbibed emulsions in porous medium. Moreover, this provided increasing of viscosity and a change of emulsions behaviour as a liquid.
\end{abstract}

\section{Introduction}

The spontaneous imbibition is one type of liquid transport in porous structures which is driven by capillary pressure. It appears on pores walls during their wetting by a penetrating liquid, if an adhesion force of liquid molecules exceeds the force of their mutual attraction [14]. The imbibition is known as a phenomenon occurring in the natural environment, i.e. hydrological regime in a soil matrix, migration processes in geology; plants seeds imbibition with water. Nowadays, it is applied in numerous industrial spheres: in the environmental engineering (viz. oil and its derivatives recovery and absorption); in material engineering (viz. percolation of the antifungal emulsions into concrete constructions), as well as in pharmacology and medicine.

The experimental data on the spontaneous imbibition presented in literature can be divided into the following groups: study of the dependence between the process rate (i.e. mass/height change) and time [1, 5-10]; study of the relation between the characteristics of porous structure and the imbibition rate [1,7-9]; and study of the effect of the penetrating liquid characteristics on this process $[5,6]$. Majority of the represented results has been related to the imbibition with single-phase liquids [1, 2, 3, 5-10], while the issues concerning multiphase liquids, viz. emulsions, still have not been fully understood and described. It relates with the complexity of the mentioned process.

Emulsions being thermodynamically metastable, in practice can be stabilized by surfactants or solid particles
[11-14], or by means of decreasing the droplets size of the dispersed phase [15-17]. However, the addition of stabilization agents is currently the common method used for emulsion stabilization. This explains a great deal of the results regarding the influence of emulsifiers on emulsions stability, size distribution of droplets, their shape, and dispersity $[11-14,18,19]$.

The purpose of our research work is to investigate and describe the process of porous media imbibition with two-phase liquids. Concretely, this paper provides the experimental results about the influence of the emulsifier agent fraction and the dispersed phase concentrations on the imbibition process. The estimated values were change of mass and height of the imbibed emulsions as a function of time and variation of emulsions concentration versus height of their front during the imbibition process. In addition to the above, the correlation between the surfactant fraction and the viscosity of the prepared emulsions was investigated.

\section{Experimental part}

\subsection{Materials}

In these experiments, stabilized oil-in-water emulsions were used as wicking liquids, which dispersed phase behaved as a wetting one, while its continuous phase was absolutely non-wetting liquid. The studied emulsions had different dispersed phase concentrations $\varphi_{d}$, i.e. $10 \mathrm{vol} \%$,

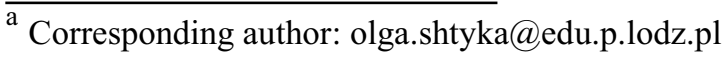


$30 \mathrm{vol} \%$, and $50 \mathrm{vol} \%$. The oily component was represented by the refined vegetable oil produced by EOL Polska Sp.z.o.o., Poland. The viscosity of pure oil was $56.3 \pm 0.46 \mathrm{mPa} \cdot \mathrm{s}$ and its density was equal to $954.0 \pm 1.7$ $\mathrm{kg} / \mathrm{m}^{3}$. The surface tension was $32.2 \pm 1.3 \mathrm{mN} / \mathrm{m}$. Ethoxylated fatty acid was used as non-ionic surfactant in fractions $\varphi_{s}$ of $1 \mathrm{vol} \%, 2 \mathrm{vol} \%$, and $5 \mathrm{vol} \%$. The commercial name of the emulsifier is Rokacet $\mathrm{O} 7$ and it is produced by PCC Exol SA, Poland.

The porous medium was represented in a form of stripes with a height $h$ of $0.016 \mathrm{~m}$ and a width $a$ of $0.035 \mathrm{~m}$. They were cut from a nonwoven polypropylene sheet. The average porosity of investigated medium was equal to 0.93 and the mean thickness was $1.9 \times 10^{-3} \mathrm{~m}$. This material belonged to the type of oleophilic sorbent. According to experimental data, the sorption capacity of vegetable oil $(\eta \sim 53.1 \mathrm{mPa} \cdot \mathrm{s})$ equaled $24 \mathrm{~g} / \mathrm{g}$, while pure water was not wicked. The investigated polypropylene sorbent was obtained from Sintac ${ }^{\mathbb{C}}$-Polska Sp.z o.o., Poland.

\subsection{Procedure of the experiment}

The dependence between the concentration of the added surfactant and the rate of the imbibition process, changes of the dispersed phase concentration and viscosity of imbibed emulsion versus height were investigated experimentally. Each of the prepared polypropylene stripes was dipped into a beaker with $200 \mathrm{ml}$ of the emulsion [20]. The contact square of material surface was $1.4 \times 10^{-4} \mathrm{~m}^{2}$.

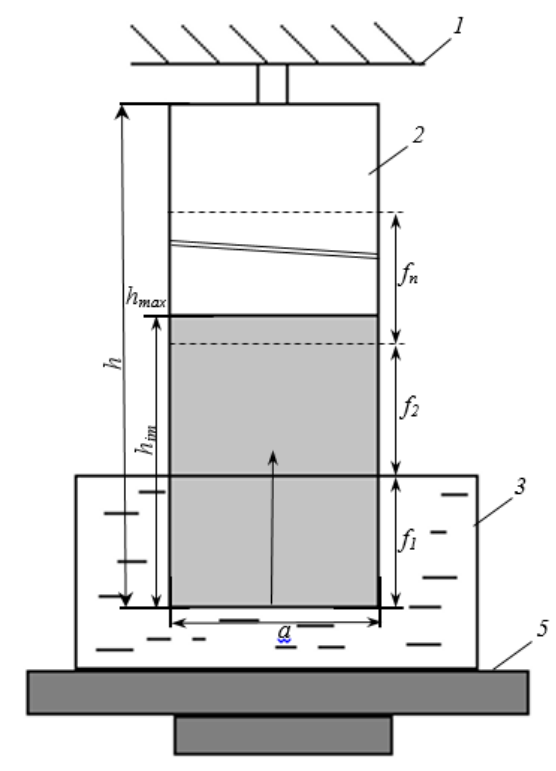

Figure 1. Scheme of the used equipment: 1 - fixator of a porous material; 2 - stripe of a porous material; 3 - reservoir with a liquid; 5 - balance.

The changes of the height of an imbibed liquid front, i.e. $h_{i m}$, and mass $m_{i m}$ versus time $t_{i m}$ were followed till the time when their values became unalterable, and it was denoted as $t_{\max }$. After that, a sorbent stripe was removed from the beaker and its soaked part was cut into fragments $f_{n}$. The size of each fragment was of a width of
$0.035 \mathrm{~m}$ and a height of $0.02 \mathrm{~m}$ (Figure 1). Each sample was put into a ceramic dish and after that, the imbibed emulsion was washed out with distilled water.

In obtained mixture the concentration of the dispersed phase was measured by means of the nephelometrical method using of the optical analyzer TurbiscanTM LAB (Formulaction, France). This method has been reported elsewhere [21].

\subsection{Analytical methods}

The density of the prepared emulsions was measured by means of the picnometric method. The viscosity changes related to increasing of the surfactant and inner phase concentrations were determined using a rotational viscometer Rheometer RC 20 (Rheotec, Germany). The shear rate values varied and were in a range of 80-140 $1 / \mathrm{s}$. The surface tension was measured with a tensiometer KRÜSS K100, Germany.

All experiments were conducted at a temperature of $23 \pm 1^{\circ} \mathrm{C}$ and atmospheric pressure.

\section{Results and discussion}

\subsection{Viscosity changes vs surfactant concentration increasing}

According to the obtained data, the increase of a surfactant concentration caused changes of viscosity of the investigated emulsion and their behavior as a liquid. As shown in Figure 2, the flow curves can be approximated by a straight line in case of all emulsions with a dispersed phase concentration of $10 \%$ and $30 \%$ emulsions with $\varphi_{s} \leq 2$ vol\%. Thus, it means that the investigated disperses behaved as Newtonian liquids, while other emulsions, mainly with higher concentrations of inner phase, behaved as non-Newtonian liquids.

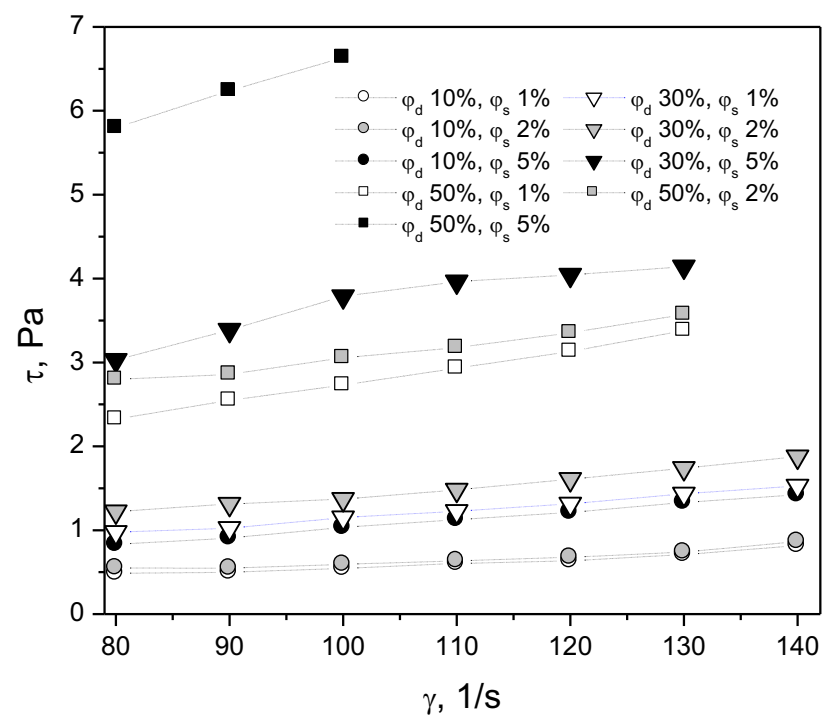

Figure 2. Dependence between shear stress and the surfactant concentration at different shear rates. 


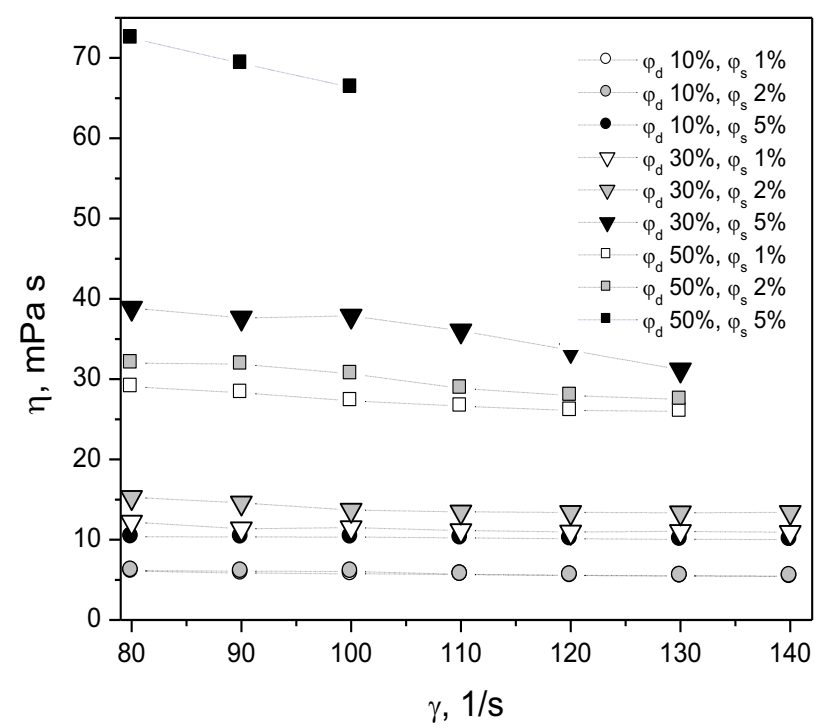

Figure 3. Changes of viscosity versus increasing of the surfactant concentration at different shear rate.

The same conclusions were made based on viscosity data analyzing. Its values were constant at different shear rate for all $10 \%$ emulsion and $30 \%$ emulsions with $\varphi_{s}=1$ and $2 \mathrm{vol} \%$. Others behaved as the non-Newtonian shear thinning liquids, thus, increasing of the shear rate caused decreasing of the viscosity value. For $30 \%$ emulsion it reduced from $38.9 \mathrm{mPa} \cdot \mathrm{s}$ for $\gamma=801 / \mathrm{s}$ till $31.2 \mathrm{mPa} \cdot \mathrm{s}$ for $\gamma=130 \mathrm{1} / \mathrm{s}$ and for another one, viscosity was $72.5 \mathrm{mPa} \cdot \mathrm{s}$ at shear rate $801 / \mathrm{s}$, while for $10001 / \mathrm{s}$, it decreased to $66.4 \mathrm{mPa} \cdot \mathrm{s}$ (Figure 3).

\subsection{Changes of imbibition rate vs increasing of surfactant concentration}

The preliminary experiments showed that the process of porous media imbibition with emulsions depended on a fraction of added surfactant and this revealed to be a variation of the height and weight of imbibed liquids. The results concerning obtained maximal mass of the liquids imbibed in the non-submerged part of sorptive media, are presented in Table 1.

Table 1. Maximal values of mass, $[\mathrm{kg}]$.

\begin{tabular}{|c|c|c|c|}
\hline \multirow{2}{*}{$\begin{array}{c}\text { Fraction of } \\
\text { surfactant, } \\
\varphi_{s}\end{array}$} & \multicolumn{3}{|c|}{ The dispersed phase concentration, $\varphi_{d}$} \\
\cline { 2 - 4 } & $10 \mathrm{vol} \%$ & $30 \mathrm{vol} \%$ & $50 \mathrm{vol} \%$ \\
\hline $1 \mathrm{vol} \%$ & 0.0015 & 0.0009 & 0.0004 \\
\hline $2 \mathrm{vol} \%$ & 0.0016 & 0.0012 & 0.0011 \\
\hline $5 \mathrm{vol} \%$ & 0.0024 & 0.0022 & 0.0012 \\
\hline
\end{tabular}

The increase of emulsifier fraction caused a raise of the imbibed emulsion mass in all investigated cases. For $50 \%$ emulsions, the value of imbibed mass was 3 times higher at $\varphi_{s}=5 \mathrm{vol} \%$ comparing to the emulsion with the lowest surfactant fraction, i.e. 1 vol\%. Such ratio was equaled to 2.5 for $30 \%$ emulsion and 1.6 for $10 \%$, respectively. The same tendency was observed for the changes of height of the imbibed emulsions. As an example, the results for $10 \%$ and $50 \%$ emulsions are plotted in Figure 4-5.

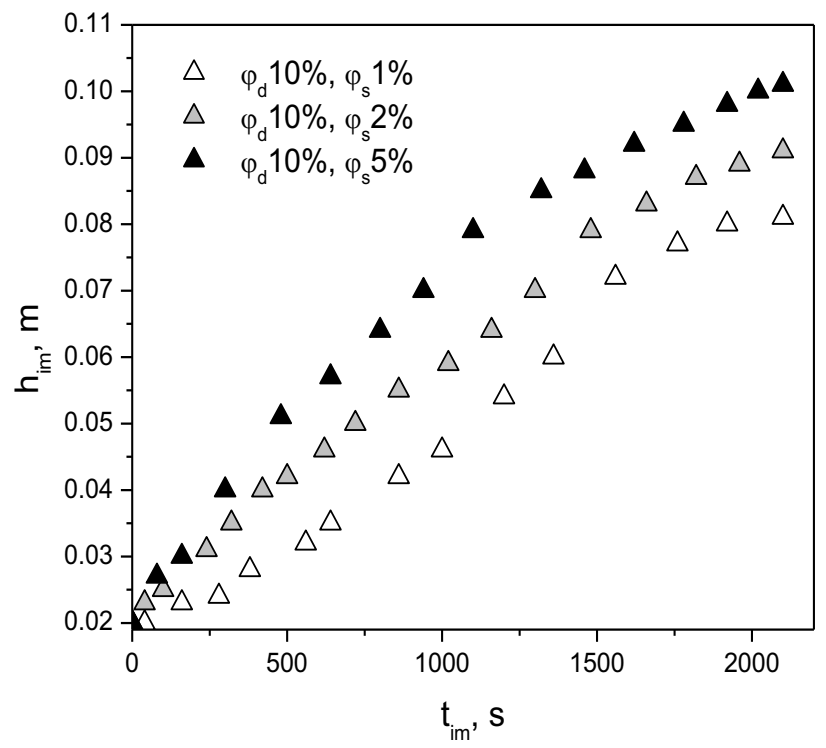

Figure 4. Change of an imbibed liquid height vs time for emulsions with different fractions of surfactant $\left(\varphi_{d}=10 \mathrm{vol} \%\right)$.

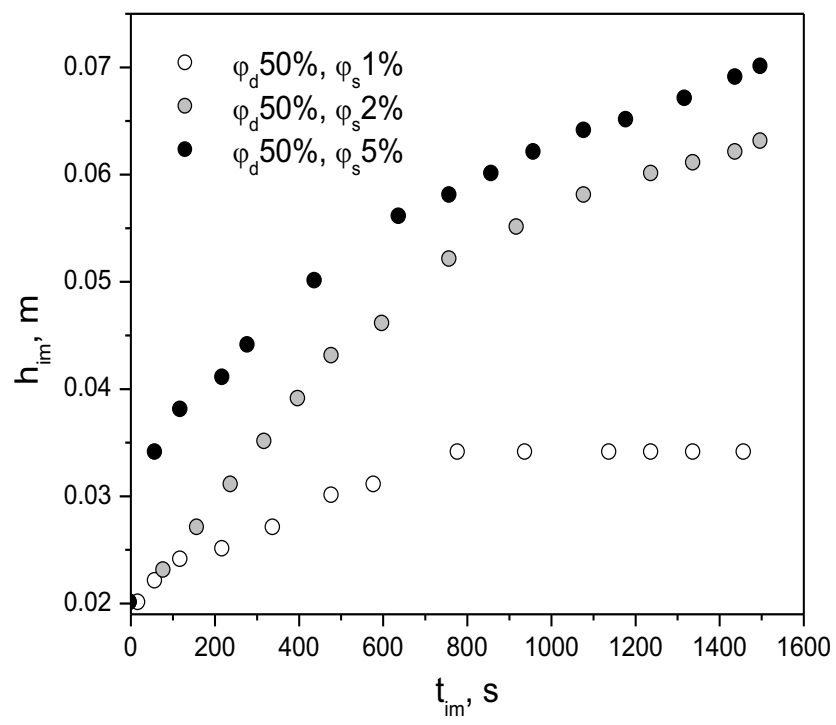

Figure 5. Change of an imbibed liquid height vs time for emulsions with different fractions of surfactant $\left(\varphi_{d}=50 \mathrm{vol} \%\right)$.

In case of $10 \%$ emulsions, it increased from $0.081 \mathrm{~m}$ $\left(\varphi_{s}=1 \mathrm{vol} \%\right)$ to $0.101 \mathrm{~m}\left(\varphi_{s}=5 \mathrm{vol} \%\right)$, i.e. by $25 \%$. For $30 \%$ emulsions, the difference of the fronts heights was $0.02 \mathrm{~m}(\sim 30 \%)$. Whereas, in case of $50 \%$ emulsions, the increasing of surfactant fraction till $5 \mathrm{vol} \%$ caused rising of the height $h_{i m}$ by $75 \%$, viz. from 0.04 to $0.07 \mathrm{~m}$.

\subsection{Changes of the dispersed phase concentrations vs height}

The changes of the dispersed phase concentrations versus the height of an imbibed emulsion are shown in Figure 68. On the graphs, the vertical axis represents the normalized values of the dispersed phase concentrations which were calculated as a ratio $\varphi_{i m} / \varphi_{d}$ where $\varphi_{i m}$ is the obtained experimentally value of the concentration in an 
imbibed emulsion and correspondingly, $\varphi_{d}$ is defined as the initial concentration of the dispersed phase.

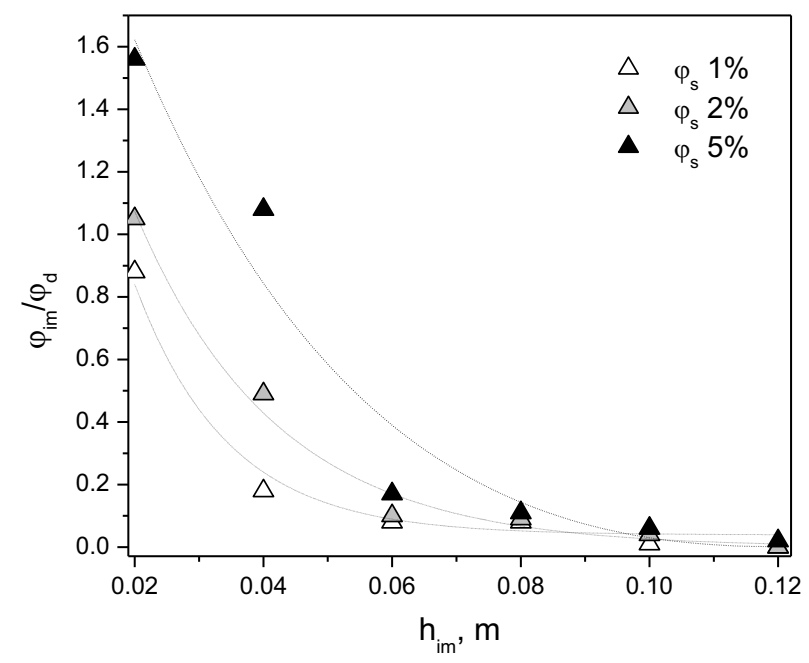

Figure 6. Changes of the emulsions concentrations vs height for different fractions of surfactant $\left(\varphi_{d}=10 \mathrm{vol} \%\right)$.

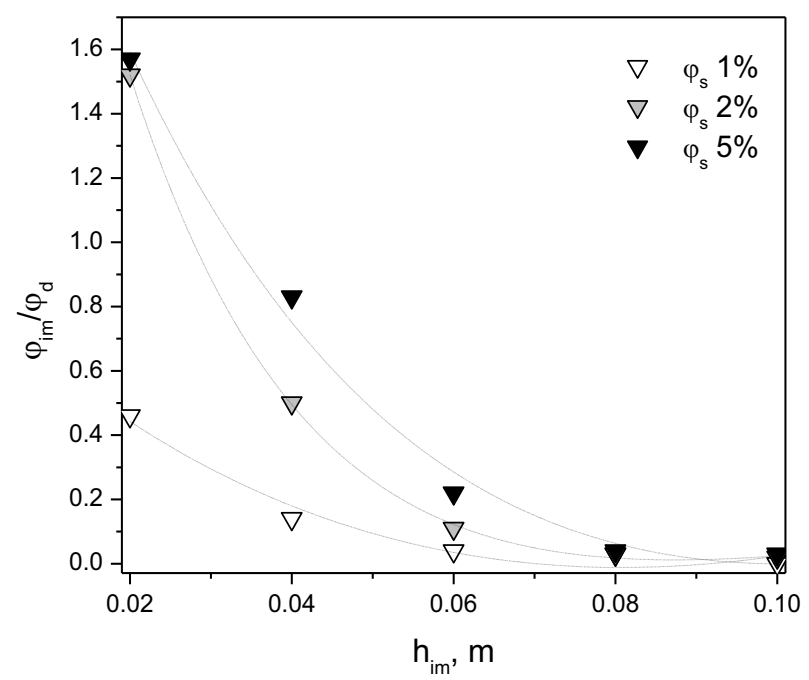

Figure 7. Changes of the emulsions concentration vs height for different fractions of surfactant $\left(\varphi_{d}=30 \mathrm{vol} \%\right)$.

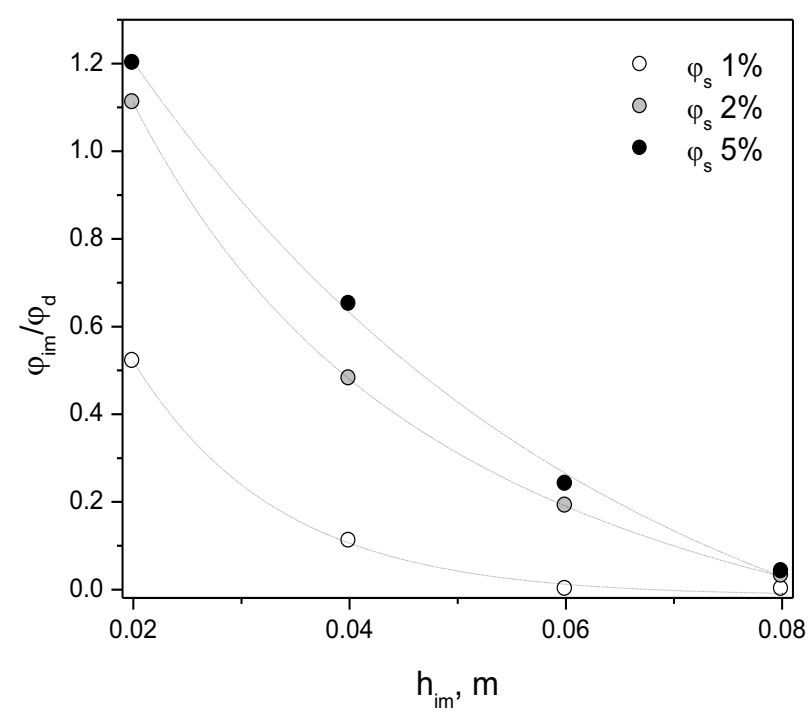

Figure 8. Changes of the emulsions concentration vs height for different fractions of surfactant $\left(\varphi_{d}=50 \mathrm{vol} \%\right)$.
In all investigated cases, the obtained concentrations of dispersed phase were lower in case of $\varphi_{s}=1 \mathrm{vol} \%$, and consequently, the highest were observed for $\varphi_{s}=5 \mathrm{vol} \%$.

At the height $h_{i m} \leq 0.02 \mathrm{~m}$, the obtained concentration of the imbibed emulsion exceeded its initial value $\varphi_{d}$, but only when surfactant was added in amount of $2 \mathrm{vol} \%$ and $5 \mathrm{vol} \%$. However, at $h_{i m}=0.08 \mathrm{~m}$, the obtained values of the dispersed phase concentration were almost identical for emulsions with different surfactant fractions, but the same content of oil phase (Figure 6-8).

In case of $30 \%$ and $50 \%$ emulsions, the curves describing the concentration changes, beside $\varphi_{s}=1 \mathrm{vol} \%$, were relatively close to each other (Figure 7-8). It proves that for emulsions with a higher dispersed phase concentration $\left(\varphi_{d} \geq 30 \mathrm{vol} \%\right)$, the increasing of surfactant concentration caused little effect on the investigated process.

\subsection{Changes of the emulsion viscosity vs height}

As previously mentioned, the influence of added surfactant was estimated from the point of their stabilization and the decrease rate of deemulgation process. However, the surfactants influence also on the emulsion ability to penetrate through porous structure.

The obtained results concerning changes of the emulsion viscosity versus height are shown in Figure 911.

In case of surfactant concentration of $1 \mathrm{vol} \%$, the tendency of the viscosity decreasing was observed for all investigated emulsions (Figure 9). At $h_{i m} \leq 0.02 \mathrm{~m}$, the obtained values of viscosity were approximately the same as the initial ones. For emulsion with $\varphi_{d}=10 \mathrm{vol} \%$, the front of imbibed liquid was higher, but the viscosity was the lowest. However, the maximal value of viscosity was observed for $50 \%$ emulsion at $h_{i m} \leq 0.04 \mathrm{~m}$.

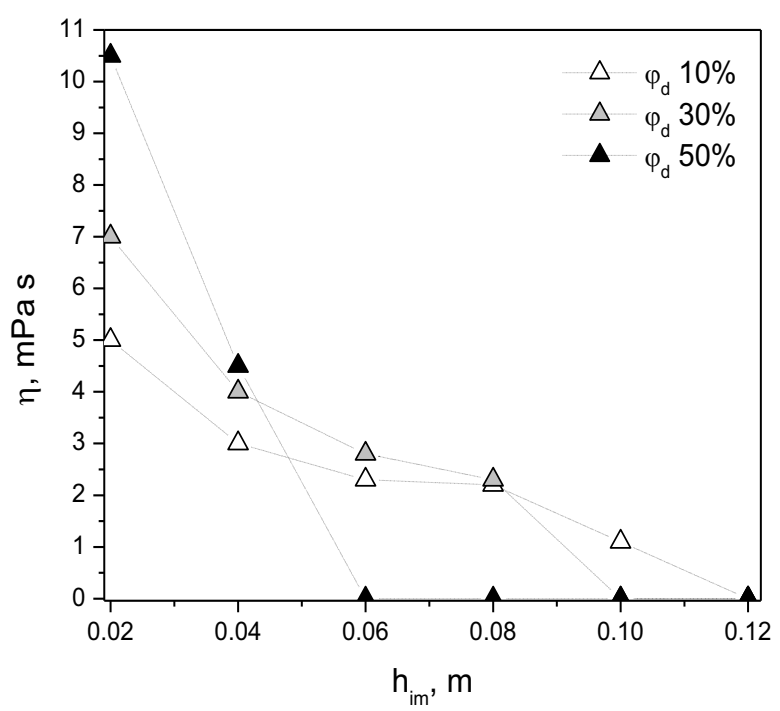

Figure 9. Changes of the viscosity vs height for different values of the dispersed phase concentration $\left(\varphi_{s}=1 \mathrm{vol} \%\right)$.

As shown in Figure 10, at $h_{i m} \leq 0.02 \mathrm{~m}$, the obtained viscosity values were a few higher in comparison with initial data for all emulsions with $\varphi_{s}=2$ vol $\%$. However, 
there was a tendency of the viscosity decrease at $h_{\text {im }} \geq 0.02 \mathrm{~m}$

As in the previous case, the maximal values of viscosity were obtained for the emulsion with $\varphi_{d}=50$ vol $\%$, but for $h_{i m} \leq 0.08 \mathrm{~m}$, consequently the lowest were observed for $10 \%$ at the height less than $0.10 \mathrm{~m}$ (Figure $10)$.

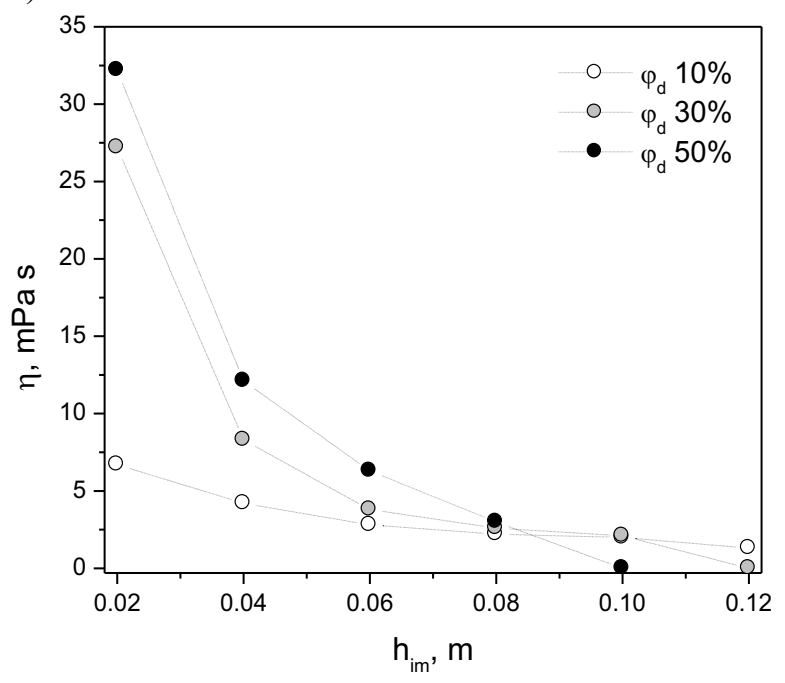

Figure 10. Changes of the viscosity vs height for different values of the dispersed phase concentration ( $\varphi_{s}=2$ vol\%).

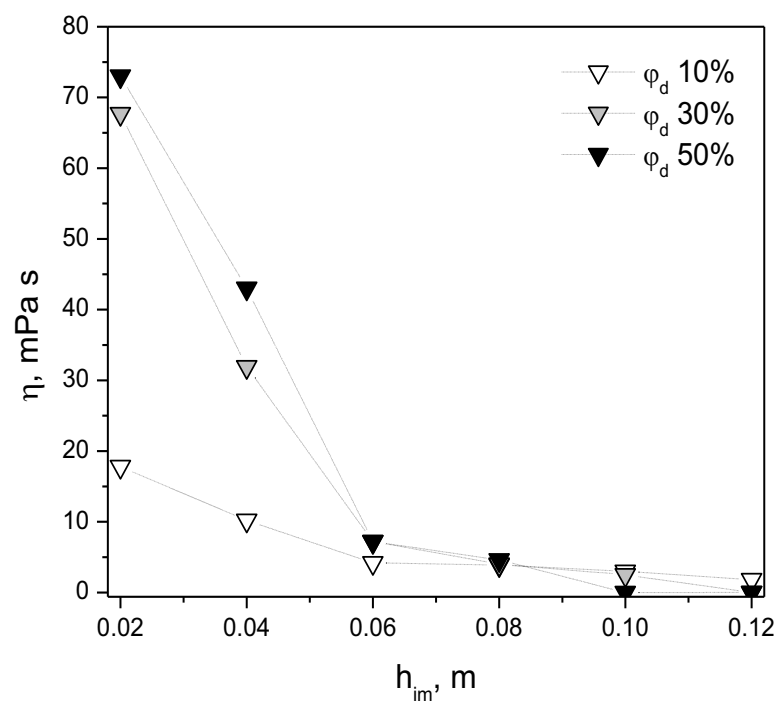

Figure 11. Changes of the viscosity vs height for different values of the dispersed phase concentration $\left(\varphi_{s}=5 \mathrm{vol} \%\right)$

The effect of the surfactant addition in an amount of 5 vol\% was exceeding of viscosity values obtained for the initial emulsions. This phenomenon was observed at height $h_{i m} \leq 0.02 \mathrm{~m}$ and the main reason can be the increase of the phase concentration as shown in Figure 68.

However, at $h_{i m} \geq 0.02 \mathrm{~m}$, the viscosity of an imbibed emulsion decreased with increasing of their front height and it was observed for all investigated emulsions.

\section{Conclusions}

To sum up, the amount of the surfactant influenced the rheological properties of the investigated liquids.

For example, the emulsions with a low concentrated dispersed phase, i.e. $\varphi_{d}<30 \mathrm{vol} \%$, and surfactant volume fraction of $\varphi_{s}<2 \mathrm{vol} \%$ behaved as Newtonian liquids, while at $\varphi_{d} \geq 30 \mathrm{vol} \%$ and $\varphi_{s} \geq 2 \mathrm{vol} \%$, they exhibited properties of non-Newtonian liquids.

Moreover, the process of porous structures imbibition with surfactant-stabilized emulsions depended on both the initial concentration of the inner phase and the surfactant fraction. Firstly, it revealed increasing of the weight and height of the imbibed emulsions with a raise of surfactant fraction.

In contrast, their decreasing was observed simultaneously with an increase of the dispersed phase concentration. The obtained results also allowed to conclude that at $h_{i m} \geq 0.02 \mathrm{~m}$, the inner phase concentration and viscosity of imbibed emulsion decreased versus height of its front in a porous medium.

\section{Acknowledgment}

The study was funded in framework of the Foundation Program for Young Scientists in Lodz University of Technology (Grant No. 501/10-34-2-1015).

\section{References}

1. Masoodi R., Pillai K.M., AIChE, 56, 9 (2010)

2. Alava M., Dube M., Rost M., Adv. Phys. 53 (2004)

3. Zhmud B.V., Tiberg F., Hallstensson K. J., Coll. Interf. Sci., 228 (2000)

4. Chatterjee P. K., Gupta B. S. Textile Science and Technology, 13 (2002)

5. Xue H.T., Fang Z.N., Yang Y., Huang J.P., Zhou L.W., Chem. Phys. Lett., 432 (2006)

6. Siebold A., Nardin M., Schultz J., Walliser A., Oppliger M., Coll. Surf., 161 (2000)

7. Hamraoui A., Nylander T., J. Coll. Interf. Sci., 250, 2 (2002)

8. Masoodi R., Pillai K.M., Varanasi P.P., AIChE J. 53, 11 (2007)

9. Dang-Vu T., Hupka J., Phys. Pr. Miner. Proc., 39 (2005)

10. Cai J., Hu X., Standnes D. C., You L., Coll. Surf. A: Physicochem. Eng. Aspects, 414 (2012)

11. Blink B.P., Lumson S.O., Langmuir, 16 (2000)

12. Bibette J., Leal-Calderon, Curr. Opin. Colloid Interface Sci., 1 (1996)

13. Blink B.P., Current Opinion Coll. Interf. Sc., 7 (2002)

14. Leal-Calderon F., Schmitt V., Curr. Opin. Colloid Interface Sci., 13 (2008)

15. Rahn-Chiquea K., Puertas A.M., Romero-Canob M.S., Rojasa C., Urbina-Villalbaa G., Adv. Coll. Interf. Sci., 178 (2012)

16. Tadros T., Izguierdo P., Esquena J., Solans C., Adv. Coll. Interf. Sci., 108 (2004)

17. Gutierrez J.M., Gonzalez C., Maestro A., Sole I., Pey C.M., Nolla J., Curr. Opin. Colloid Interface Sci., 13 (2008)

18. Binks B.P., Jhonny A.R., Langmuir, 23, 7 (2003) 
19. Behrend O., Ax K., Schubert H., Ultrasonics Sonochem., 7 (2000)

20. Sęk, J., Shtyka O., Szymczak K., J. Environ. Eng. Landsc., 23, 2 (2015)
21. Lemarchand C., Couvreur P., Vauthier C., Costantini D., Gref R., Int. J. Pharm., 254 (2003) 